\title{
MicroRNA expression in pediatric intracranial ependymomas and their potential value for tumor grading
}

\author{
ŠIMON CIPRO ${ }^{1}$, MARIE BELHAJOVÁ ${ }^{2}$, TOMÁŠ ECKSCHLAGER ${ }^{2}$ and JOSEF ZÁMEČNÍK ${ }^{1}$ \\ Departments of ${ }^{1}$ Pathology and Molecular Medicine, and ${ }^{2}$ Pediatric Hematology and Oncology, \\ Second Faculty of Medicine, Charles University and University Hospital Motol, 15006 Prague 5, Czech Republic
}

Received February 28, 2018; Accepted October 26, 2018

DOI: $10.3892 / \mathrm{ol} .2018 .9685$

\begin{abstract}
Intracranial ependymoma represents one of the most common pediatric central nervous system malignancies, and exhibits a wide range of clinical behavior from relatively indolent lesions to highly malignant anaplastic ependymomas. Due to the heterogeneous nature of this disease there is lack of prognostic markers, which would reliably predict the outcome of patients. MicroRNAs (miRNAs) have emerged as important molecules in cancer biology during past decade; however, very little is known about their role in ependymomas. The aim of the present study was to evaluate expression of miRNAs in archived formalin-fixed paraffin-embedded (FFPE) samples of pediatric intracranial ependymomas. The expression of miRNAs were examined in 29 samples of ependymoma and we observed that miR-135a-3p, miR-137, miR-17-5p, miR-181d and let-7d-5p were upregulated. In addition, a significantly higher expression of miR-203a was detected in Grade III tumors suggesting its possible use as a prognostic or diagnostic marker. The present study also demonstrated that storage of (FFPE) ependymoma samples for $>20$ years did not result in a deterioration of miRNAs. The present findings broaden the presently available knowledge regarding miRNA expression in ependymomas and provide further evidence for the employment of miRNA analysis as a supplementary method for the morphological assessment of ependymoma samples.
\end{abstract}

\section{Introduction}

Ependymoma represents one of the most common pediatric intracranial malignancies constituting approximately $9 \%$ of all pediatric brain tumors $(1,2)$. Despite their relatively high incidence, there is a lack of appropriate prognostic factors besides extent of surgical resection (3).

Correspondence to: Dr Šimon Cipro, Department of Pathology and Molecular Medicine, Second Faculty of Medicine, Charles University and University Hospital Motol, V Uvalu 84, 15006 Prague 5, Czech Republic

E-mail: simon.cipro@gmail.com

Key words: ependymoma, microRNA, miR-203, miR-137, let-7d
Previous studies have shown that it is hard to predict patient outcome based on histology and/or genetics of the tumor. One of the underlying reason is a heterogeneous nature of the disease $(4,5)$.

The traditional histopathological grading also remains controversial and fails to predict clinical behavior and outcome of the disease (6). The extent of surgical resection still remains the most important predictor of overall survival and time-to-relapse (7). The need for new biomarkers for better diagnosis, grading and management of disease progression is evident.

MicroRNAs are short non-coding molecules that have a major impact on gene expression (8). More than 1,500 miRNAs have been identified in human genome (9). miRNAs have also been shown to play various roles in cancer. Mainly, they have emerged as important regulators in human carcinogenesis by affecting expression of tumor-suppressor genes and oncogenes $(10,11)$.

Although our understanding of the role of miRNAs has substantially improved in recent years, not much attention has been given to the role of miRNAs in ependymomas $(12,13)$. The aim of our work is to broaden our current knowledge of miRNAs' role in pediatric intracranial ependymomas and elucidate their possible use as a new biomarkers and prognostic factors.

\section{Materials and methods}

Patients and samples. A total of 29 formalin-fixed paraffin-embedded (FFPE) specimens of ependymomas were collected retrospectively from the archives of Department of Pathology and Molecular Medicine, University Hospital Motol, Prague, Czech Republic. FFPE specimens of ependymoma were obtained from patients treated between years 1985-2017. All patients were under 18 years of age with median age of 6 years (Table I). The histological grade was evaluated independently by two observers according to WHO criteria. Our cohort consisted of 14 grade II tumors and 15 grade III tumors. This study was approved by Ethical committee of Second Faculty of Medicine, Charles University and University hospital Motol, Prague, Czech Republic.

FFPE tissue from plexus choroideus and lining of lateral brain ventricles from 5 patients (median age 11 years) who 
died from non-brain-related illnesses was used as a control group.

RNA extraction. Total RNA was extracted from FFPE blocks from five to eight $10 \mu \mathrm{m}$-thick tissue sections. The RecoverAll ${ }^{\mathrm{TM}}$ Total Nucleic Acid Isolation kit for FFPE (Ambion; Thermo Fisher Scientific, Inc., Waltham, MA, USA) according to manufacturer's instructions was used. Total RNA quantity and quality was evaluated using a spectrophotometer (Nanodrop ND-1000, Thermo Fisher Scientific, Inc.).

Relative quantification of miRNA expression. Total RNA extracted as described above was converted to cDNA by reverse transcription using miRNA specific TaqMan ${ }^{\circledR}$ primer and TaqMan ${ }^{\circledR}$ MicroRNA Reverse Transcription kit (Applied Biosystems; Thermo Fisher Scientific, Inc.) according to manufacturer's instructions. qPCR was performed using TaqMan ${ }^{\circledR}$ Individual miRNA assays (14) for miR-135a-3p, miR-137, miR-17-5p, miR-181d, miR-203a, let-7d-5p, RNU48 and miR-596 (Applied Biosystems; Thermo Fisher Scientific, Inc.) and TaqMan ${ }^{\circledR}$ Universal PCR Master Mix according to manufacturer's instructions. All qPCR reactions were performed in triplicate on 96-well plate.

Statistical analysis. For all miRNA quantification experiments cycle threshold $(\mathrm{Ct})$ values were normalized against the expression levels of RNU48. $\Delta \mathrm{Ct}$ values and fold changes were calculated using DataAssist Software v. 3.01 (Applied Biosystems; Thermo Fisher Scientific, Inc.). An unpaired Student's t-test was performed to compare the $\Delta \mathrm{Ct}$ values. P-values of fold changes were adjusted using Benjamini-Hochberg false discovery rate method using DataAssist Software. Statistical significance for all experiments was attributed if $\mathrm{P} \leq 0.05$.

\section{Results}

Concentration and purity of RNA isolated from long-time archived samples is equal with more recent specimens. Subset of samples from our study was archived in (FFPE) blocks for 25-30 years. In order to verify if the oldest samples in our study are suitable for further analysis we assess purity and concentration of those specimens.

Furthermore, we performed individual RQ-qPCR assay for RNU48 to see variation in threshold cycles. Table II shows that neither RNA concentration nor the ratio of absorbance at 260 and $280 \mathrm{~nm}$ (A260/280 nm) does significantly differ between samples of different age. Mean value of A260/280 nm is 1.95 (SD 0.02). Also Ct values of RNU48 was not impaired by the age of FFPE blocks. These results indicate that even the oldest FFPE specimens in our study were suitable for further analysis.

miRNAs are differentially expressed in ependymomas. We performed individual assays for group of miRNAs and the results revealed that miR-135a-3p, miR-137, miR-17-5p, miR-181d and let-7d-5p are differentially expressed in ependymomas when compared to control group $(\mathrm{P}<0.05)$. All of those miRNAs were overexpressed (Fig. 1, Table III). The miR-203 was found to be overexpressed with the highest difference as compared to control group (fold change 46.3), but the result
Table I. Clinicopathological features of ependymomas included in the present study.

\begin{tabular}{lcc}
\hline Age & $\%$ & Number \\
\hline$<4$ years & 34.48 & 10 \\
4-15 years & 55.17 & 16 \\
$>15$ years & 10.34 & 3 \\
Grade 2 & 48.28 & 14 \\
Grade $3^{\mathrm{a}}$ & 51.72 & 15 \\
\hline
\end{tabular}

${ }^{\mathrm{a}}$ WHO criteria $(4,5)$.

was not statistically significant $(\mathrm{P}=0.08)$. Our results also indicate that the miR-34c belonging to the tumor suppressor family miR-34 is $(15,16)$ slightly downregulated in ependymoma samples although the difference was not statistically significant ( $R Q$ 0.9, $\mathrm{P}=0.75$ ).

MiR-203a is overexpressed in grade III tumors. Statistical analysis was performed in order to find differences in expression of miRNAs between grade II and grade III ependymomas. We found miR-203a to have higher expression in grade III ependymomas (Fig. 2, Table III) suggesting its possible role in distinguishing more aggressive tumors. Others miRNAs that are differentially expressed in tumor samples when compared to normal controls did not show statistically significant differences between grade II and grade III tumors.

\section{Discussion}

Ependymomas exhibit wide range of biological behavior extending from low-grade lesions to highly malignant tumors. Prognostic stratification based solely on morphology or immunohistochemistry remains elusive and brings only poor results in regards to patient outcome.

In this study we aim at studying expression levels of various miRNAs using individual qPCR assays in order to identify new prognostic and/or diagnostic markers of ependymomas. We have chosen miRNAs based on results of our preliminary experiment (data not shown) where more than 20 miRNAs were involved. Out of this preliminary experiment we have chosen seven miRNAs which were showing differential expression between normal and ependymoma samples or at least trend towards significance for this study.

We have identified miR-135a-3p, miR-137, miR-17-5p, miR-181d and let-7d-5p to be differentially expressed in ependymoma samples. To our knowledge this is the first report of overexpression of miR-137 in pediatric intracranial ependymoma. The let- $7 \mathrm{~d}$ has been previously described to act both as a tumor-suppressor and oncogene $(17,18)$. In ependymomas it was previously shown (12) that let-7d correlates with overall survival. However, we have not found significant difference in let-7d expression between grade II and grade III samples. This result underlines the fact that miRNA are pluripotent molecules and their role in tumorigenesis in given tumor type needs to be elucidated by more studies especially in tumors of genetically heterogeneous nature. 
Table II. Comparison of RNA quality and quantity between samples of different ages.

\begin{tabular}{|c|c|c|c|c|c|c|}
\hline \multirow[b]{2}{*}{ Sample age } & \multicolumn{2}{|c|}{ Concentration } & \multicolumn{2}{|c|}{ A260/280 } & \multicolumn{2}{|c|}{ Ct RNU48 } \\
\hline & Mean & SD & Mean & SD & Mean & SD \\
\hline $28-30$ years & $187 \mathrm{ng} / \mu \mathrm{l}$ & 164 & 1.95 & 0.073 & 23.89 & 0.88 \\
\hline $2-5$ years & $171 \mathrm{ng} / \mu 1$ & 103 & 1.97 & 0.04 & 22.63 & 0.43 \\
\hline $1-2$ years & $173 \mathrm{ng} / \mu 1$ & 65 & 1.94 & 0.04 & 23.01 & 0.14 \\
\hline
\end{tabular}

SD, standard deviation; Ct, cycle threshold; RNU48, small nucleolar RNA, C/D Box 48-used for normalization.

Table III. Table presenting the up- vs. downregulation of miRNAs expression in normal vs. disease samples and grade III vs. grade II cohort.

\begin{tabular}{lccccc}
\hline & \multicolumn{2}{c}{$\mathrm{N}$ vs. Ep } & & \multicolumn{2}{c}{ G3 vs. G2 } \\
\cline { 2 - 3 } \cline { 5 - 6 } Assay & Fold change & P-value & & Fold change & P-value \\
\hline miR-135a-3p & 4.0 & 0.01 & & 1.0 & 0.15 \\
miR-137 & 13.0 & 0.01 & & 0.8 & 0.10 \\
miR-17-5p & 3.7 & 0.01 & & 0.8 & 0.50 \\
miR-181d & 5.6 & 0.001 & & 0.6 & 0.08 \\
miR-203a & 46.3 & 0.08 & & 6.6 & 0.01 \\
34c & 0.9 & 0.75 & & 1.0 & 0.40 \\
let-7d-5p & 3.6 & 0.01 & & 1.0 & 0.06 \\
\hline
\end{tabular}

$\mathrm{N}$, control samples; Ep, ependymoma samples; G2, Grade 2; G3, grade 3 .

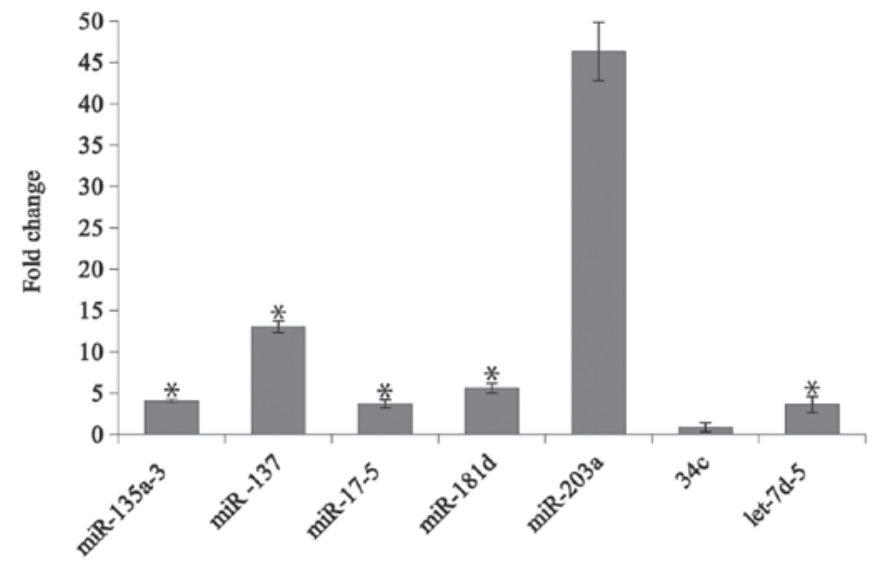

Figure 1. Graph illustrating the expression of different miRNAs in ependymoma samples when compared with a control group, the height of the column thus indicates the number of times the expression in the tumor samples is higher than in control samples. The values in the Y-axis are of fold changes based on DataAssist software. Error bars indicate standard deviation of experiments performed in triplicate. FFPE tissue from plexus choroideus and lining of lateral brain ventricles from 5 patients who died from non-brain-related illnesses were used as a control group. "P $<0.05$. miRNA, microRNA.

Tumor grading in ependymoma reveals high interindividual variability (19). This fact calls for new markers which would make distinguishing between low-grade and high-grade lesion

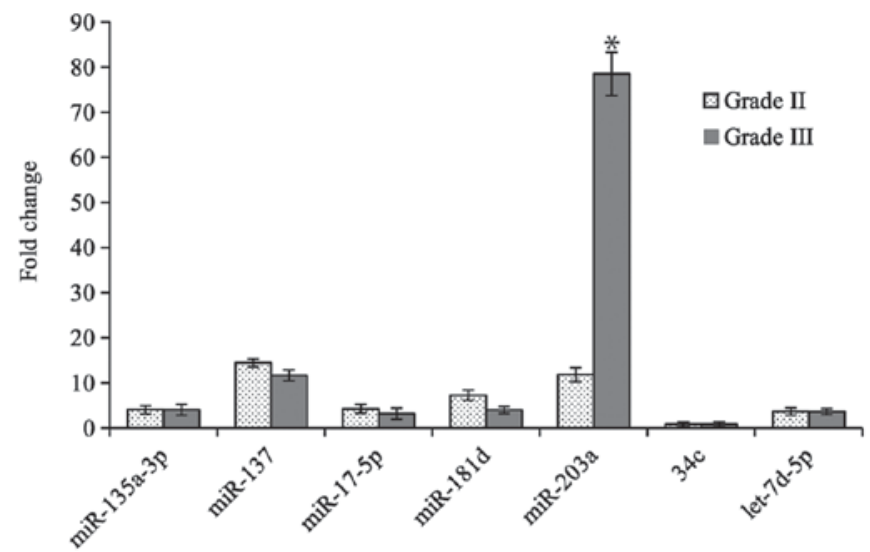

Figure 2. Graph illustrating the expression of different miRNAs in Grade II and Grade III tumors when compared with a control group, the height of the column thus indicates the number of times the expression in the tumor samples is higher than in control samples. The values in the Y-axis are of fold changes based on DataAssist software. Error bars indicate standard deviation of experiments performed in triplicate. FFPE tissue from plexus choroideus and lining of lateral brain ventricles from 5 patients who died from non-brain-related illnesses were used as a control group. ${ }^{*} \mathrm{P}<0.05$. miRNA, microRNA.

more convenient. We have demonstrated increased expression of miR-203a in grade III tumors compared to grade II tumors. In recent years there has been considerable interest in miR-203. This miRNA located on chromosome 14q32.33 was shown to have aberrant expression in many types of cancers. However, the role of miR-203 in various cancer types is not straightforward. It was previously reported that overexpression of miR-203a in glioblastoma (20) increased apoptosis of cancer cells thus acting as tumor-suppressor gene. Similarly, it was shown that miR-203 upregulation inhibits epithelial-mesenchymal transition in glioblastoma cells (21). miR-203a also suppress hepatocellular carcinoma tumorigenesis by targeting HOXD3 via EGFR-related cell signaling pathways (22). On the other hand, some studies clearly indicated significant correlation between the elevated miR-203 expression and poor overall survival in colorectal adenocarcinoma $(23,24)$ or pancreatic carcinoma (25). miR-203a was also described as a predictor of a poor prognosis in renal cell carcinoma as its silencing inhibits cell proliferation, migration and induces apoptosis (26). Despite relatively common incidence of ependymomas and increased evidence of miRNAs role in various types of cancer, not much attention has been given to role of miRNAs in ependymoma (27). It was shown that miR-17-5p, miR19a-3p 
and miR-106b-5p differentiate between grade II and III ependymomas (12). Margolin-Miller et al demonstrated that high expression of miR-124-3p in ependymomas significantly correlated with the lower progression-free survival (28).

Our observations of miR-203 expression in ependymomas are in contrast with previous findings (13) which shown that lower expression of miR-203 in ependymoma patients correlated to a trend to develop recurrences. Some evidences suggest that miR-203 might not have sufficient power to predict overall or event-free survival of patient with different types of cancer (29). It was shown that methodology employed in assuming miR-203 expression as well as the ethnicity of a patients might influence the correlation of miR-203 expression and clinical outcome of the patient (24). This could be one of the explanations of discrepancies and needs to be further elucidated.

It was previously shown that PCR amplification results from fresh-frozen material and FFPE samples are similar (30). Routinely archived FFPE samples in pathological departments are therefore suitable for retrospective analysis of miRNAs expression. We have demonstrated that FFPE samples of ependymoma archived for more than 25 years still provide high yields of RNA of excellent purity.

In conclusion, we have shown several miRNAs to be differentially expressed in ependymoma and outlined the role of miR-203. These findings may add to a growing body of literature on our understanding of miRNA expression and their role in ependymoma biology.

\section{Acknowledgements}

Not applicable.

\section{Funding}

This study was supported by The Charles University Grant Agency, grant no. GAUK190315.

\section{Availability of data and materials}

The datasets used and analyzed during this study are available from the corresponding author on reasonable request.

\section{Authors' contributions}

All authors read and approved the final manuscript. ŠC contributed to evaluation of tumor grade, for RNA extraction, quantification of miRNAs and writing of the manuscript, $\mathrm{MB}$ contributed to RNA extraction, quantification of miRNAs, TE contributed to the project design and writing of manuscript, JZ contributed to evaluation of tumor grade, the project design and writing of manuscript. All authors read and approved the final manuscript.

\section{Ethics approval and consent to participate}

This study was approved by Ethical committee of Second Faculty of Medicine, Charles University and University hospital Motol, Prague, Czech Republic. Due to the retrospective nature of this study the right to informed consent was waived.

\section{Patient consent for publication}

Not applicable.

\section{Competing interests}

The authors declare that they have no competing interests.

\section{References}

1. Gupta A and Dwivedi T: A simplified overview of World Health Organization classification update of central nervous system tumors 2016. J Neurosci Rural Pract 8: 629-641, 2017.

2. PDQ Pediatric Treatment Editorial Board: Childhood ependymoma treatment $\left(\mathrm{PDQ}^{\circledR}\right)$ : Health professional version, 2002.

3. Zamecnik J, Snuderl M, Eckschlager T, Chanova M, Hladikova M, Tichy $\mathrm{M}$ and Kodet R: Pediatric intracranial ependymomas: Prognostic relevance of histological, immunohistochemical, and flow cytometric factors. Mod Pathol 16: 980-991, 2003.

4. Hübner JM, Kool M, Pfister SM and Pajtler KW: Epidemiology, molecular classification and WHO grading of ependymoma. J Neurosurg Sci 62: 46-50, 2018.

5. Pajtler KW, Witt H, Sill M, Jones DT, Hovestadt V, Kratochwil F, Wani K, Tatevossian R, Punchihewa C, Johann P, et al: Molecular classification of ependymal tumors across all CNS compartments, histopathological grades, and age groups. Cancer Cell 27: 728-743, 2015.

6. Pajtler KW, Mack SC, Ramaswamy V, Smith CA, Witt H, Smith A, Hansford JR, von Hoff K, Wright KD, Hwang E, et al: The current consensus on the clinical management of intracranial ependymoma and its distinct molecular variants. Acta Neuropathol 133: 5-12, 2017.

7. Tihan T,Zhou T,Holmes E, Burger PC, Ozuysal S and Rushing EJ: The prognostic value of histological grading of posterior fossa ependymomas in children: A Children's Oncology Group study and a review of prognostic factors. Mod Pathol 21: 165-177, 2008.

8. Hayes J, Peruzzi PP and Lawler S: MicroRNAs in cancer: Biomarkers, functions and therapy. Trends Mol Med 20: 460-469, 2014.

9. Bentwich I, Avniel A, Karov Y, Aharonov R, Gilad S, Barad O, Barzilai A, Einat P, Einav U, Meiri E, et al: Identification of hundreds of conserved and nonconserved human microRNAs. Nat Genet 37: 766-770, 2005.

10. Vannini I, Fanini F and Fabbri M: Emerging roles of microRNAs in cancer. Curr Opin Genet Dev 48: 128-133, 2018.

11. Filipowicz W, Bhattacharyya SN and Sonenberg N: Mechanisms of post-transcriptional regulation by microRNAs: Are the answers in sight? Nat Rev Genet 9: 102-114, 2008.

12. Zakrzewska M, Fendler W, Zakrzewski K, Sikorska B, Grajkowska W, Dembowska-Bagińska B, Filipek I, Stefańczyk Ł and Liberski PP: Altered MicroRNA expression is associated with tumor grade, molecular background and outcome in childhood infratentorial ependymoma. PLoS One 11: e0158464, 2016.

13. Costa FF, Bischof JM, Vanin EF, Lulla RR, Wang M, Sredni ST, Rajaram V, Bonaldo Mde F, Wang D, Goldman S, et al: Identification of microRNAs as potential prognostic markers in ependymoma. PLoS One 6: e25114, 2011.

14. Chen C, Ridzon DA, Broomer AJ, Zhou Z, Lee DH, Nguyen JT, Barbisin M, Xu NL, Mahuvakar VR, Andersen MR, et al: Real-time quantification of microRNAs by stem-loop RT-PCR. Nucleic Acids Res 33: e179, 2005.

15. Wiggins JF, Ruffino L, Kelnar K, Omotola M, Patrawala L, Brown D and Bader AG: Development of a lung cancer therapeutic based on the tumor suppressor microRNA-34. Cancer Res 70: 5923-5930, 2010.

16. Misso G, Di Martino MT, De Rosa G, Farooqi AA, Lombardi A, Campani $\mathrm{V}$, Zarone MR, Gullà $\mathrm{A}$, Tagliaferri $\mathrm{P}$, Tassone $\mathrm{P}$ and Caraglia M: Mir-34: A new weapon against cancer? Mol Ther Nucleic Acids 3: e194, 2014.

17. Boyerinas B, Park SM, Hau A, Murmann AE and Peter ME: The role of let-7 in cell differentiation and cancer. Endocr Relat Cancer 17: F19-F36, 2010.

18. Kolenda T, Przybyła W, Teresiak A, Mackiewicz A and Lamperska KM: The mystery of let-7d-a small RNA with great power. Contemp Oncol (Pozn) 18: 293-301, 2014.

19. Leeper H, Felicella MM and Walbert T: Recent advances in the classification and treatment of ependymomas. Curr Treat Options Oncol 18: 55, 2017. 
20. Yang CH, Wang Y, Sims M, Cai C, He P, Häcker H, Yue J, Cheng J, Boop FA and Pfeffer LM: MicroRNA203a suppresses glioma tumorigenesis through an ATM-dependent interferon response pathway. Oncotarget 8: 112980-112991, 2017.

21. Liao H, Bai Y, Qiu S, Zheng L, Huang L, Liu T, Wang X, Liu Y, $\mathrm{Xu}$ N, Yan X and Guo H: MiR-203 downregulation is responsible for chemoresistance in human glioblastoma by promoting epithelial-mesenchymal transition via SNAI2. Oncotarget 6 : 8914-8928, 2015.

22. Wang L, Sun H, Wang X, Hou N, Zhao L, Tong D, He K, Yang Y, Song T, Yang J and Huang C: EGR1 mediates miR-203a suppress the hepatocellular carcinoma cells progression by targeting HOXD3 through EGFR signaling pathway. Oncotarget 7: 45302-45316, 2016.

23. Schetter AJ, Leung SY, Sohn JJ, Zanetti KA, Bowman ED, Yanaihara N, Yuen ST, Chan TL, Kwong DL, Au GK, et al: MicroRNA expression profiles associated with prognosis and therapeutic outcome in colon adenocarcinoma. JAMA 299: 425-436, 2008

24. Bovell LC, Shanmugam C, Putcha BD, Katkoori VR, Zhang B, Bae S, Singh KP, Grizzle WE and Manne U: The prognostic value of microRNAs varies with patient race/ethnicity and stage of colorectal cancer. Clin Cancer Res 19: 3955-3965, 2013 .
25. Greither T, Grochola LF, Udelnow A, Lautenschläger C, Würl P and Taubert H: Elevated expression of microRNAs 155, 203, 210 and 222 in pancreatic tumors is associated with poorer survival. Int J Cancer 126: 73-80, 2010.

26. Hu G, Lai P, Liu M, Xu L, Guo Z, Liu H, Li W, Wang G, Yao X, Zheng $\mathrm{J}$ and $\mathrm{Xu} \mathrm{Y}$ : miR-203a regulates proliferation, migration, and apoptosis by targeting glycogen synthase kinase- $3 \beta$ in human renal cell carcinoma. Tumour Biol 35: 11443-11453, 2014.

27. Birks DK, Barton VN, Donson AM, Handler MH, Vibhakar R and Foreman NK: Survey of MicroRNA expression in pediatric brain tumors. Pediatr Blood Cancer 56: 211-216, 2011

28. Margolin-Miller Y, Yanichkin N, Shichrur K, Toledano H, Ohali A, Tzaridis T, Michowitz S, Fichman-Horn S, Feinmesser M, Pfister SM, et al: Prognostic relevance of miR-124-3p and its target TP53INP1 in pediatric ependymoma. Genes Chromosomes Cancer 56: 639-650, 2017.

29. Liang Y, Yang W, Zhu Y and Yuan Y: Prognostic role of microRNA-203 in various carcinomas: Evidence from a meta-analysis involving 13 studies. Springerplus 5: 1538, 2016.

30. Siebolts U, Varnholt H, Drebber U, Dienes HP, Wickenhauser C and Odenthal M: Tissues from routine pathology archives are suitable for microRNA analyses by quantitative PCR. J Clin Pathol 62: 84-88, 2009. 